\title{
Article
}

\section{Decision Tree Analysis as a Supplementary Tool to Enhance Histomorphological Differentiation when Distinguishing Human from Non-human Cranial Bone in both Burnt and Unburnt States: A feasibility study}

Simmons, Tal Linda lleen, Goodburn, B and Singhrao, Simarjit Kaur Available at http://clok.uclan.ac.uk/11877/

Simmons, Tal Linda Ileen, Goodburn, B and Singhrao, Simarjit Kaur ORCID: 0000-0001-9573-5963 (2016) Decision Tree Analysis as a Supplementary Tool to Enhance Histomorphological Differentiation when Distinguishing Human from Non-human Cranial Bone in both Burnt and Unburnt States: A feasibility study. Medicine, Science and the Law, 56 (1). pp. 36-45. ISSN 0025-8024

It is advisable to refer to the publisher's version if you intend to cite from the work. http://dx.doi.org/10.1177/0025802415589776

For more information about UCLan's research in this area go to http://www.uclan.ac.uk/researchgroups/ and search for < name of research Group>.

For information about Research generally at UCLan please go to http://www.uclan.ac.uk/research/

All outputs in CLoK are protected by Intellectual Property Rights law, including Copyright law. Copyright, IPR and Moral Rights for the works on this site are retained by the individual authors and/or other copyright owners. Terms and conditions for use of this material are defined in the policies page. 
Title: Decision Tree Analysis as a Supplementary Tool to Enhance Histomorphological Differentiation when Distinguishing Human from Non-human Cranial Bone in both Burnt and Unburnt States: A feasibility study

Short Title: Decision Tree Analysis for Histomorphological Differentiation

Authors: Simmons $\mathrm{T}^{1 *}$, Goodburn $\mathrm{B}^{2}$, Singhrao $\mathrm{SK}^{3}$

${ }^{* 1}$ Department of Forensic Science, Virginia Commonwealth University, Richmond, VA 23284 USA

${ }^{2}$ School of Forensic and Investigative Sciences, University of Central Lancashire, Preston, PR1 2HE, U.K.

${ }^{3}$ Oral \& Dental Sciences Research Group, School of Medicine and Dentistry, University of Central Lancashire, Preston, PR1 2HE, U.K.

\section{*Corresponding author:}

Dr Tal Simmons

Tel: 1-804-828-8420

Email: tsimmons@vcu.edu 


\begin{abstract}
This feasibility study was undertaken to describe and record the histological characteristics of burnt and unburnt cranial bone fragments from human and non-human bones. Reference series of fully mineralised, transverse sections of cranial bone, from all variables and specimen states were prepared by manual cutting and semi-automated grinding and polishing methods. A photomicrograph catalogue reflecting differences in burnt and unburnt bone from human and non-humans was recorded and qualitative analysis was performed using an established classification system based on primary bone characteristics. The histomorphology associated with human and non-human samples was, for the main part, preserved following burning at high temperature. Clearly, fibro-lamellar complex tissue subtypes, such as plexiform or laminar primary bone, were only present in non-human bones. A decision tree analysis based on histological features provided a definitive identification key for distinguishing human from non-human bone, with an accuracy of 100\%. The decision tree for samples where burning was unknown was $96 \%$ accurate, and multi-step classification to taxon was possible with $100 \%$ accuracy. The results of this feasibility study, strongly suggest that histology remains a viable alternative technique if fragments of cranial bone require forensic examination in both burnt and unburnt states. The decision tree analysis may provide an additional, but vital tool to enhance data interpretation. Further studies are needed to assess variation in histomorphology taking into account other cranial bones, ontogeny, species and burning conditions.
\end{abstract}

Key words: Decision tree analysis, histology, human, non-human, burnt, unburnt, bone 


\section{Introduction}

Decision tree analysis is a powerful tool to identify human/non-human origin of fragmentary cranial bones in burnt and unburnt states. Identifying a fragmentary bone specimen with ambiguous morphology as definitively originating from a human or non-human source is challenging, especially if the specimen is rendered unfit for DNA or albumin analyses. It is not uncommon for a perpetrator to set a fire in an attempt to destroy evidence, including that found on a corpse. Thus, small burnt bone fragments are encountered in forensic cases, and when this happens, they can be extremely difficult to identify and interpret. Furthermore, if the fragment is of a suspected human “flat” bone origin, lacking anatomical landmarks, accuracy of reporting its exact origins becomes even more challenging. The identification of such specimens, by experts in the field may be critical to the case, especially if no other recognisable body parts are present.

Histology has been documented previously as a viable option when determining whether a bone is of human or non-human origin [1-9]. In this respect, histology can be used to examine de-mineralised bone and teeth on a routine basis. Our own experience in handling burnt bone in the laboratory is that it easily fractures under any applied pressure. Examining such fragile forensic or archaeological specimens requires that their histological analysis be performed in their native mineralised state, supported within resin media; de-calcification in the laboratory should be avoided as it would render burnt specimens unfit for processing into various embedding media. This study was conducted to address a gap in the literature and was initiated subsequent to the recovery of purportedly human cranial bone fragments in a forensic case, which recently featured in a high profile abduction and murder trial in the UK $[10,11]$. As histolomorphological analysis can be subjective, the novel application of a decision tree analysis to supplement the existing histomorphological analysis was explored. Therefore, the aim of this preliminary study was to examine whether tree analysis could be 
used as an additional tool to help to accurately describe the recorded histological characteristics of human and non-human cranial bone fragments and determine the animal origin in both unburnt and burnt states. This was accomplished by leaving bones in their original state (i.e bone specimens were not de-calcified in the laboratory), as burnt bone is expected to lack its matrix proteins that support mineralization in situ. Discriminating between human and non-human bone rests on the microanatomy and size of specific structures in the tissue being examined. Thus, the developed methods rely on both qualitative and quantitative analyses [7] provided the bony fragment retains specific histological characteristics such as the plexiform bone or osteon banding [2].

\section{Materials and Methods}

Approval for this study was obtained before commencing laboratory investigations in which all experimental procedures met the ethical guidelines of our academic institute (Ref no. 045). Cellmark Forensic Services (UK) also approved the study with their donated specimens (G20397932, dated 29 May 2013). Cranial bone specimens selected for this study were from adult humans $(\mathrm{N}=6)$, adult dog, adult sheep, adult badger, and both adult and juvenile pigs (all $\mathrm{N}=1$ ). Of the six human bone specimens, three were exhumed in 1997 in Milan, Italy and were unclaimed finds. The Milan skulls were of known sex and age, originally in possession of Prof. C. Cattaneo (Milan, Italy), who donated them to Cellmark Forensic Services (UK) for scientific research in 2006. The UK, Human Tissue Act of 2004, does not apply to the Milan skulls as, following their exhumation in 1997, they have remained an unclaimed entity and were donated and imported into the UK for research in 2006. During the specimen collection period of this study, three additional fragmentary dry human cranial bone specimens became available, two modern anatomical parietal fragments, one each from Cellmark and the University of Central Lancashire. An archaeological specimen, from the parietal fragment, was 
also examined, which was donated by Cellmark. All the specimens used in this study are presented in Table 1; human samples were form either the right or left parietal bone. The crania from non-human (dog, sheep, badger and pig) origins were all derived from the University of Central Lancashire, zoological comparative osteology collection. All specimens were sampled similarly to the human skulls, although the site of the bone sampled in the non-human specimens varied, based on ease of access and variable robustness, the non-human specimens were removed from the frontal, parietal and occipital regions of the skull.

\section{$\underline{\text { Sample preparation }}$}

Following their removal from the original skulls, the bones used in this study were not chemically fixed (e.g. formalin), dehydrated or infiltrated with resin for bone histology techniques $[12,13]$. Instead, all cranial bone samples were prepared following the currently established methods described by Cattaneo et al. [2, 3] for forensic bone histomorphological investigations. Samples from each specimen (both human and non-human) were paired, with one set burnt while and the other was left unburnt.

\section{Unburnt bone}

Panels of cranial bone approximately $6 \mathrm{~cm} \mathrm{x} 4 \mathrm{~cm}$ were cut from the crania using a small circular power saw (Dremel, UK) and then trimmed into pieces measuring $2 \mathrm{~cm} \mathrm{x} 1 \mathrm{~cm}$. The edges of all fragments were lightly sanded, cleaned and dried prior to bonding onto glass microscope slides with Pertex mounting medium (Cellpath, UK Ltd). Once firmly held on the glass slide, the specimen was fully covered in more Pertex and allowed to cure for at least 3-7 days prior to preparing sections using the grinding and polishing techniques.

\section{Burnt samples}


Bone specimens were burnt in a bench top furnace (Carbolite, UK) for 20 minute at 700 ${ }^{\circ} \mathrm{C}$ to simulate house and car fire situations [2]. As the burnt bone specimens were extremely fragile, they were directly embedded in Araldite CY1300GB mixed with Araldite HY951 (Robnor Resins Ltd., UK) in a 9:1 ratio as described by Cattaneo et al., [2, 3]. The specimen was gently lowered into the resin mixture and minor adjustments were made to make sure the bone was in the desired orientation for subsequent grinding and polishing. More resin mixture was poured into the mold to fully cover the specimen and was then left to cure for a week at room temperature. Following curing, the specimen block was removed from the mold and mounted onto a glass slide using Pertex (see section above on unburnt bone).

\section{Preparation of ground sections (all bones)}

In this study, the manual cutting and grinding methods described by Cattaneo et al., [2, 3] were initially employed. However, in view of the labour intensive protocol and a large number of blocks that needed grinding and polishing, it was decided to switch to a semiautomated commercial grinder and polisher (Ecomet 250, Buehler UK) as described elsewhere [13]. To avoid damaging the fragile specimen, the tissue block was ground under cooling conditions using graded and adhesive backed sand paper circles (240-800 grit) (Buehler Krautkramer, UK) where 240 is course grit and 500-800 is medium grit for grinding. To prevent over-grinding, the section was monitored under the light microscope (Eclipse E200, Nikon UK) to the required thickness (approximately $120 \mu \mathrm{m}$ ). The specimen was then carefully polished by using 1200 and 2500 fine grit sand paper (Buehler, UK) to allow cyto-architectural evaluation by light microscopy. This reduced the overall section thickness to around $100 \mu \mathrm{m}$ and allowed visualisation by light microscopy. All sections from each of the burnt and unburnt bones were examined without the introduction of any stains. All sections were examined using a Nikon Eclipse E200 microscope and imaged using the Nikon DS-L2 v.441 software. 
A photo-catalogue and the classification table from Cuijpers et al., [3] were adapted and merged in order to provide a visual key for assigning tissue types. Initially, a qualitative assessment as described by Cuijpers [4, 5], was undertaken to identify features that reliably distinguish between specimens of non-human and human origin. The classification system adopted in this study is shown in Table 2. The system used to categorize histomorphology was related to the full width of the cranial bone transverse section including the trabecular bone. The abbreviations, codes and classification used in the table are cross referenced with Cuijpers [5]. The slides were examined in a specific orientation, where the ectocranial table was always located at the top of the image. Photomicrographs of the three designated zones in each transverse section, namely ectocranial, intermediate, and endocranial, were recorded.

\section{$\underline{\text { Statistical analysis }}$}

A decision tree analyses was performed using the statistical software package R [14]. A data matrix was created as the basis of the analysis; this matrix summarised the presence or absence of the tissue types observed in all the histology slides for each taxon, in both burnt and unburnt states. A tree model is a simple and computationally powerful way of providing structure to the data and, in particular, the hierarchy among many potential explanatory variables [15]. The analyses employed herein are classification trees for replicate data and are used to separate human from non-human histomorphological states based on the presence or absence of the 25 possible explanatory variables (tissue types) observed. The best key is, by definition, the one with the lowest classification error, i.e. the one that misclassifies the fewest cases.

\section{Results}


A continuum of primary bone structure types were identified from all specimens under the light microscope whereby overlap of the fibro-lamellar complex bone structures was also apparent. The longitudinal or reticular nature of the Haversian canals was also observed together with numerous micro fractures in sections prepared from unburnt and/or burnt states.

\section{Unburnt specimens}

The transverse-sections of the bone in their native state from all five species (Fig. 1) displayed two layers of dense cortical bone and two layers of trabecular bone. The microanatomy of the specimens demonstrated primary lamellar and Haversian features characteristic of true bone. Many of the osteons observed under the microscope appeared in their oblique plane. No fibro-lamellar complex bone (1f) was observed in any of the cranial specimens examined. Haversian canal type was predominantly of longitudinal type (HC1). In some unburnt bone sections, linear arrangements of up to 11 primary osteons (1c5) running parallel to ectocranial or endocranial edges were observed. Other examples of osteon banding were seen with secondary osteons, but only a maximum of four osteons appeared to align (2a1b). There was one example of both primary and secondary osteons forming a single osteon band.

\section{Dog parietal bone}

The histomorphology of the canine parietal bone was predominantly of the fibrous bone types with some primary lamellar and Haversian systems (Fig. 1a). The fibro-lamellar complex types were varied but reticular bone (1f3) was observed in all specimens. Secondary osteons

exhibited both longitudinal and reticular Haversian canals. Trabecular bone and also some smaller primary osteons and banding were noted (1c5) in which the primary osteons outnumbered the secondary osteons. 


\section{Sheep frontal bone}

The histomorphological structures of frontal bone of sheep varied, showing a complicated and convoluted pattern with primary lamellar, fibro-lamellar complex and Haversian systems (Fig. 1b). Reticular fibro-lamellar (1f3) and irregular Haversian bone was also present in abundance. The microanatomy resembled that of human bone in some respect to the trabecular bone morphology. For example, the osteons present in the trabecular bone were abundant with variable sizes of Haversian canals as well as the shape, distribution and size of the bony spicules and vacuoles, but the reticular nature of sheep - bone tissue helped to distinguish it from human bone. There were also similarities between the sheep and the dog bony structures, because of the reticular nature of these bones.

\section{Badger parietal bone}

One characteristic feature of the badger bone was that it was considerably thinner than the cranial bone from all the other species examined. Transverse-sections lacked regions of trabecular bone in this species (Fig. 1c). The histomorphology was mainly of the reticular fibrolamellar complex type (1f3) in which there was a thick layer of lamellar bone (1a) within the endocranial edge. Marked primary and secondary osteon banding was observed. The reticular fibrous tissue aided the distinction of badger cranial bone from that of the human bone. Only a few secondary osteons were observed in these sections of bone and they were difficult to distinguish from primary osteons.

\section{Adult pig parietal bone}


The adult pig parietal fragment exhibited a type of fibro-lamellar complex bone which is typically plexiform (1f2), reticular (1f3) or pseudo-laminar (1f1/1a-c) (Fig. 1d). The unburnt bone sections revealed few scattered secondary osteons and lamellar tissue types. Only one example of primary osteon banding (1f5) was recorded. Fibro-lamellar complex bone types created a thick layer in the ectocranial zone. The central band of the cross-section was trabecular with a homogenous pattern. Primary osteons outnumbered secondary osteons and large resorption bays were visible in the endocranial zone giving the edge a serrated appearance (not shown).

\section{Adult pig occipital bone}

The histomorphology of the adult pig occipital bone differed to that of the parietal bone in the same animal. Plexiform layer (1f2) was absent but laminar types were present (1f1, 1f1/1a-c). Trabecular tissue resembled the form seen in the parietal bone sections along with large resorption bays in the endocranial zone.

\section{Immature pig frontal bone}

In the immature pig and the adult pig frontal bone, the histomorphology was predominantly of the fibro-lamellar type with little Haversian bone. Primary lamellar bone types were visible in the unburnt samples mainly as scattered primary osteons in trabecular spicules. Non-vascular lamellar structures (1a) appeared as a growth layer within plexiform in one sample. Plexiform (1f2) tissue was found in all samples as a thick band at the ectocranial surface (Fig. 1e). Internal to this was a band of laminar (1f1) or pseudo-laminar bone (1f1/1ac). Some osteon banding (1f5) was identified in unburnt material. 
Developing fibro-lamellar bone was visible at ectocranial and endocranial surfaces. The histomorphology of the occipital bone was similar to that seen in the frontal bone. Plexiform bone type was dominant. The variety and orientation of fibro-lamellar complex types differed to those observed in the frontal bone. All specimens displayed at least one fibro-lamellar complex type. Net and brick like plexiform structures were seen in the ectocranial band.

\section{Human (anatomical and Milan) parietal bones}

Cross-sections displayed the same overall tissue architecture seen in human anatomical specimens, but with larger osteocyte lacunae. It was difficult to distinguish primary reticular canals (1b3) from reticular Haversian canals (HC3). All samples had longitudinal Haversian canals (HC1) with many osteons appearing in the transverse and oblique plane (Fig. 1f). No fibro-lamellar complex bone was observed and patches of fibrous bone (1d) were rare. Some primary (1c5) and secondary (2a1b) osteon banding was observed. No more than five secondary osteons, aligned parallel to the ectocranial edge. Altogether 27 primary osteons were recorded, and no more than seven aligned with each other.

\section{Human (archaeological) parietal bone}

All transverse-sections of the human archaeological bone were poorly preserved and, consequently, their microscopic detail proved difficult to interpret. The tissue sections appeared degraded, and presented a lack of identifying bony tissue structures. Furthermore, fungal infiltrations secondary to post burial taphonomic processes, were present in the poorly preserved bony structure and obscured any histomorphological features. Hence, these bones were of little value to the identification criteria being applied and tested in this study before and after burning.

\section{Burnt specimens}


The native burnt tissue pieces were very fragile and easily crumbled when handling them during processing in Araldite. During sectioning, micro fractures were particularly abundant in tissue sections of burnt specimens at $700{ }^{\circ} \mathrm{C}$ for twenty minutes. The bony sections demonstrated heat damage and had an overall opaque appearance when examined under the light microscope (Fig. 2).

\section{Dog parietal bone}

Burnt parietal bone from dog origins showed defined scattered secondary osteons separated by dark opaque areas. Overall, the microanatomy of the burnt samples was adequately recognizable as being characteristic of canine (dog) origin (Fig 2a). Osteons were densely packed in the area of the sagittal crest.

\section{Sheep frontal bone}

In tissue sections from burnt samples of the sheep cranial bone, lamellae types were not identifiable. Microscopically, there was extensive charring and opaqueness about the section leading to lack of clear structural definition (Fig. 2b).

\section{Badger parietal bone}

The tissue sections from burnt bone demonstrated that the microscopic bony structure types in the badger parietal bone were difficult to interpret due to the opaque patches and uniform heat damaged areas. These areas reflected the distribution of fibrous tissue seen in unburnt samples. Despite heat induced changes the overall distinctive pattern for badger bone remained intact (Fig. 2c).

\section{Adult pig parietal bones}


The burnt bone structures observed in the ground sections of the adult pig parietal bone exhibited fibro-lamellar complex tissue types. Uneven heat damage was evident as there were areas of the lamellar bone that were preserved with areas that were poorly preserved throughout the burnt specimens (Fig. 2d).

\section{Adult pig occipital bones}

The sections from the burnt adult pig occipital bone showed that all of the histological definition of lamellae in the bone was missing. In addition, the fibro-lamellar complex type was not seen in unburnt sections of bone (1f7). Conversely there were areas in the tissue section that remained undamaged by heat such as the circumferences of secondary osteons. Characteristic fibro-lamellar complex structure types on the bone were visible in all 31 burnt samples. Charring and heat damage were also observed in the intermediate and endocranial zones.

\section{Immature pig frontal and occipital bones}

Sections from bone revealed that structure types such as the Harversian and lamellar types, normally observed in immature pig were not discernible following burning. Trabecular bone was homogeneous with some granularity and charring within the section (Fig. 2e).

\section{Human Milan skull parietal bones}

There were no significant differences in microanatomy among the three Milan crania except for '026', in which reticular primary vascular canals were more abundant. Indications of damage from burning in the human Milan skulls were noted in the ectocranial and endocranial zones by the presence of dark homogenous patches. 


\section{Human (anatomical) parietal bones}

Overall, no fibrous bone was observed in the burnt specimens from human bone. Radial simple vascular canals (1b4) and primary osteon banding (1c5) was observed in all of the burnt specimens. The parietal bone specimens from human sources displayed some lamellar and Haversian characteristics that were also noted in the ground-sections of unburnt bones (Fig. 2f).

\section{Human (archaeological) parietal bone}

Sections from the burnt human archaeological bone severely lacked bony microscopical structures see unburnt bone section above.

\section{Decision Tree analysis}

Albeit given the small sample size in this study, results of the decision tree analysis were unambiguous. This was true regardless of which cranial bone (parietal, frontal or occipital), burnt or unburnt, was sampled. For unburnt samples, it was possible to distinguish modern human from non-human cranial bone with 100\% accuracy based on the presence (nonhuman) or absence (human) of a single tissue type, 1f1a-c (pseudo-laminar and pseudo fibrolamellar complex). For burnt specimens, the presence of tissue type $1 \mathrm{~b} 3$ (reticular lamellar bone) alone indicated with $100 \%$ accuracy that the bone was human, and its absence indicated a non-human origin. In the event that it is unclear whether a bone fragment is burnt or unburnt, decision tree analysis will still classify the histological tissue slide as human or non-human with an accuracy of $96 \%$ (27/28 specimens); this process employs a two-step identification key based on both the tissue types used in the previous analyses (Fig. 3). The one specimen that misclassified was the human archaeological specimen as its preservation was compromised and, consequently, the1b3 type histomorphology could not be accurately 
assessed. Further decision tree analysis (minimum group size of two) from the entire data set, burnt and unburnt samples combined, facilitated the classification of taxon from tissue type with 100\% accuracy using a multiple step model (Fig. 4).

\section{Discussion}

Histology does not always provide definitive information for forensic evidence in legal cases but it can be used when all other primary definitive (e.g. molecular and biochemical analyses) methodological approaches are rendered useless by the state of the specimen available for forensic analysis. Origins of bone can only be determined microscopically as long as the fragment undergoing laboratory examination retains specific histological characteristics such as the plexiform bone or osteon banding [2]. These histological features are key elements to discriminating between human and non-human bone both morphologically and metrically. These features have been described using the specimen preparation methods of Cattaneo et al., [2, 3]. This is an established method of preparing fragile bony specimens for forensic analysis. However, in order to reduce micro fractures, and the trapping fine dust during the grinding and polishing steps; the methodology of Cattaneo et al., [2, 3] may eventually be superseded by acrylic resin based methodologies that involve infiltration of the resin into the tissue specimen prior to polymerisation and/or direct mounting onto the glass slide [13]. Thus, better histological preparation of the already compromised tissue specimens for clarity of the final histological presentation are also important for an accurate interpretation of bony structures from difficult forensic specimens. Given that the trends toward the Regulatory Governance of expert witnesses as well as new rules of evidence admissibility (e.g. Daubert), forensic practitioners require an additional framework for the interpretation of results from histological examination of forensic specimens that provides confidence limits and accuracy levels. 
Thus, this study was undertaken to assess species-specific variation in burnt and unburnt cranial bone histomorphology because such bony fragments are under investigated for their forensic relevance $[10,11]$. Furthermore, this feasibility study was extended to assess whether tree analysis could be used as an additional tool to accurately describe the recorded histological characteristics of human and non-human cranial bone fragments to determine the taxon in both unburnt and burnt cranial bone. The types of primary bone where the fibro-lamellar complex was intact provided the most important criteria for differentiating taxa. The absence of the fibro-lamellar complex tissue types (laminar or plexiform) and the presence of more Haversian canal systems provided features that distinguished human bone from mammalian bone, except for the one poorly preserved, archaeological specimen. The general histomorphology observed in the animal species agreed with the generalized bony tissue types already documented in long bones $[1,5,6,7,16]$. Microanatomy was used as the defining measure, e.g. 1f7 plexiform in pig samples, for non-human species-specific differences. Marked differences were also detected in the burnt samples in which osteon banding was considered to be an important tool for distinguishing specimens in non-human long bones [8].

In this study, however, the histomorphology of the cranial bones suggested that osteon banding was unreliable due to the inconsistent observation of Haversian systems. Osteon banding in non-human bone sections demonstrated better alignment of osteons than those seen in human bony tissue sections. As shown in comparable long bone studies $[1,5]$, the nature of the Haversian canal, whether longitudinal (HC1) or reticular (HC3), did not prove to be an important criterion for differentiating species. HC1 was seen in all occurrences of Haversian type tissue whilst HC3 was seen to a lower extent.

It has been suggested that a lack of orientated stress load lines throughout the cranial vault bone results in a lower alignment of osteons compared to the alignment found in long 
bones. Consequently the histology of cranial bones appears less organized than that of the long bones, making it difficult to interpret species related differences $[17,18]$. In this investigation, the secondary osteons appeared in multiple orientations across all species, except for the sagittal crest area of the dog bone in transverse sections. Since this is the only specimen examined here that has an area of directional strain, the oblique appearance of osteons may be due to the sagittal crest development as a functional rather than evolutionary reason. For example, the increase in the size of the temporalis muscle takes place during growth following forces of mastication [16]. Although it is plausible to suggest that the oblique appearance of osteons reflects a true feature of cranial bone histology, an alternate explanation could be a poor grinding technique. If uneven pressure is applied on the tissue block during the grinding and polishing of the tissue block, this can distort the plane of the grinding surface and subsequently the whole section. Under such conditions osteons will appear in their oblique plane.

This study demonstrates that tissue sections with cancellous bone often appeared opaque making histomorphological interpretation difficult. Forbes [19], has also reported that spongy, cancellous bone is readily destroyed by heat compared to the more robust compact cortical bone. Other heat induced effects noted on the cranial bones were black deposits, granularity, and micro fractures [20-22]. The cranial bone obtained from the badger was most severely damaged by heat, which could be due to its lower cross-sectional thickness compared to the cranial bones from all other species tested. Our findings closely agree with those described by Cattaneo et al., [2] for burnt bone specimens, suggesting qualitative rather than metric assessment is possible in the forensic context. The decision making tree used in this study aids that qualitative assessment following a conscious documentation of specific micro anatomical features of bone. Despite the devastating effects of burning, it is clear from this study that the underlying microanatomy remained identifiable providing the original 
sample was adequately preserved, and to a certain extent was microscopically distinguishable between the different species. The more definitive results of decision tree analysis highlight the importance of going beyond a solely qualitative morphological analysis. Often the process of identifying every tissue type present in each sample results in an overwhelming variety and distinguishing features. Deciding which of these might be the key to classification is better achieved with statistical analysis.

The morphological examination results can be greatly strengthened by decision tree analysis for rapid identification of human versus non-human bone. This study presents the idea of including decision trees to supplement the histomorphological based analysis on relatively few specimens; however, more research is required with larger sample numbers and blind testing of the identification keys presented here. Furthermore, an updated methodology for preparation of mineralized ground bone sections from fully infiltrated and resin embedded tissue blocks would provide sections of greater clarity and improve the visualization of anatomical features in bone sections. This study does provide scope for future investigations in which human from non-human cranial bone in both burnt and unburnt states should be examined using a decision tree analysis.

\section{Acknowledgements}

The authors wish to thank Professor C. Cattaneo (Università Degli Studi di Milano, Italy) and both Dr Julie Roberts and Ms Linda Ainscough (Cellmark Forensic Services, UK), for providing access to the samples from the Milan skulls. We are also grateful to Mr Peter Cross (University of Central Lancashire) for providing the non-human crania. This research received no specific grant from any funding agency in the public, commercial, or not-for-profit sectors.

\section{Conflict of interest}

The authors declare that they have no conflict of interest. 


\section{Ethical Standards}

The experiments conducted herein comply with the current UK laws and ethical clearance was granted by The University of Central Lancashire's School of Forensic and Investigative Sciences Health, Safety and Ethics Committee on 6 June 2013, ref no 045.

\section{Author's contribution}

TS performed the tree analysis and was the primary supervisor of BG for the MSc project; BG performed the laboratory based experiments, imaging and report writing as part of his M.Sc dissertation. SKS supervised BG in the laboratory and everyone jointly contributed to the manuscript preparation.

\section{Guarantor of Work}

Tal Simmons is the nominated author to be the guarantor of the work in this manuscript. 


\section{References}

[1] Brits D. Steyn M, L’Abbe,E A histomorphological analysis of human and non-human femora. Int J Legal Med 2013;doi:10.1007/soo414-013-0854-3

[2] Cattaneo C, DiMartino S, Scali S, et al. Determining the human origin of fragments of burnt bone: a comparative study of histological, immunological and DNA techniques. $J$ Forensic Sci 1999; 102:181-91

[3] Cattaneo C, Porta D, Gibelli D et al. Histological Determination of the human origin of bone fragments. J Forensic Sci 2009; 54(3):531-533.

[4] Cuijpers, S (A.G.F.M) Histological identification of bone fragments in archaeology: telling humans apart from horses and cattle. Int J Osteoarchaeol 2006;16:465-80

[5] Cuijpers, SA Distinguishing between the bone fragments of medium-sized mammals and children. A histological identification method for archaeology. Anthropol Anz 2009; 67(2):181203

[6] Martiniakova M, Crosskopf B, Omelka R, et al. Histological study of compact bone tissue in some mammals: a method of species determination. Int J Osteoarchaeol 2007;17:82-90

[7] Hillier ML, Bell LS Differentiating human from animal bone: a review of histological methods. J Forensic Sci 2007;52(2):249-63 
[8] Mulhern DM, Ubelaker DH (2001) Difference in Osteon banding between human and nonhuman bone. J Forensic Sci 2001;46(2):220-222

[9] Mulhern DM, Ubelaker DH Differentiating Human from Nonhuman Bone Microstructure. In: Crowder,C, Stout, S eds., Bone Histology: an anthropological perspective. Boca Raton: CRC Press, 2012:109-134

[10] BBC NewsApril Jones trial: Bone fragments from 'human skull' 21 May 2013. http://www.bbc.com/news/uk-wales-22596503 (last checked 9 February 2015)

[11] Crown Prosecution Service http://www.cps.gov.uk/news/latest_news/cps_statement_on_conviction_of_mark_bridger/ 30.05.2013. (last checked 2 July 2014)

[12] Singhrao SK, Müller CT, Gilbert SJ, et al. An immunofluorescence method for postembedded tissue in acrylic resin Technovit 9100 New ${ }^{\circledR}$ using fluorescein isothiocyanate secondary detection marker. Microsc Res Tech 2009; 72(7):501-6

[13] Singhrao SK, Nicholson K, Crean S Informed choices for challenging specimens when choosing methacrylate resin systems for histology. Microsc Res Tech 2012; 75(5):576-85

[14] Ripley B tree:Classification and regression trees. $\mathrm{R}$ package version 1.0-34. http://CRAN.R-project.org/package=tree, 2013 
[15] Crawley M (2002) Statistical Computing: An Introduction to Data Analysis using S-Plus. West Sussex: John Wiley \& sons, Ltd.

[16] Hidaka S, Matsumoto M, Ohsako S, et al. A histometrical study on the long bones of raccoon dogs, Nyctereutes procyonoides and badgers, Meles meles. J Vet Med Sci 1998; 60(3):323-6

[17] Cool SM, Hendrikz JK, Wood WB Microscopic age changes in the human occipital bone. J Forensic Sci 1995; 40(5):789-796

[18] Stout S Small bones of contention, In: Steadman, D, ed. $2^{\text {nd }}$ Edition. Hard Evidence: case studies in forensic anthropology. Upper Saddle River: Pearson Education, 2009: 239-47

[19] Forbes G The effects of heat on the histological structure of Bone. Police J 1941; 14(1):5060

[20] Nelson R A microscopic comparison of fresh and burned bone. J Forensic Sci 1992; 37(4):1055-60

[21] Greenlee D, Dunnell R Identification of fragmentary bone from the Pacific. J Archaeol Sci 2010; 37:957-70

[22] Castillo R, Ubelaker D, Acosta J, et al. Effects of temperature on bone tissue, Histological study of the changes in the bone matrix. Forensic Sci Int 2013; 226:33-37 


\section{Figure legends}

Fig. 1 Unstained ground sections from fully mineralised unburnt (human and non-human) cranial bone specimens showing histomorphological features that distinguish animal and human bone. The montage shows: a) dog and b) sheep (mainly lamellar); c) badger (no spongy bone); d) adult pig and e) immature pig (both plexiform bone); and f) human (primary and secondary osteons) (all scale bars $=100 \mu \mathrm{m})$

Fig. 2 Unstained ground sections from the paired burnt set shown in Figure 1. These were fully mineralised cranial bones burnt for $20 \mathrm{~min}$ at $700{ }^{\circ} \mathrm{C}$ and then directly embedded in Araldite CY1300GB epoxy resin. Despite the heat damage, the montage shows that the essential histomorphological features from a) dog, b) sheep, c) badger, d) adult pig, e) immature pig, and f) human are still recognisable (all scale bars $=100 \mu \mathrm{m}$ ).

Fig. 3 Human/Non-human decision tree diagram illustrating the two step identification key for burnt or unburnt cranial bone. The top of each step provides the tissue type and each branch represents the presence (tick) or absence $(\mathrm{X})$ of that type

Fig. 4 Taxon classification decision tree diagram illustrating a multi-step identification key for burnt or unburnt cranial bone. The top of each step provides the tissue type and each branch represents the presence (tick) or absence (X) of that type 